Int. J. Dev. Biol. 57: 61-67 (2013)

doi: $10.1387 / \mathrm{ijdb} .120098 \mathrm{ra}$

\title{
Successful whole embryo culture with commercially available reagents
}

\author{
HANNAH C. GLANVILLE-JONES, NGAI WOO and RUTH M. ARKELL* \\ Early Mammalian Development Laboratory, Research School of Biology, The Australian National University, \\ Canberra, ACT, Australia
}

\begin{abstract}
Since its development in the 1970's, whole embryo culture (WEC) has provided an important method of growing and observing murine embryos ex utero. During WEC, embryos are immersed in a combination of rat serum and cell culture media, and supplied with heat and appropriate mixtures of $\mathrm{CO}_{2}$ and oxygen that mimic growth conditions in utero. One significant factor limiting the widespread use of WEC is the perception that commercially produced rat serum is inadequate to support normal rates of embryonic growth and development. Conversely, production of serum 'in-house' is technically demanding, time-consuming and expensive. The current study aimed to identify a WEC medium comprising commercially manufactured rat serum that would produce cultured embryos of comparable standard to those grown in utero. A mixed culture medium, composed of $\mathbf{5 0} \%$ commercial rat serum and $\mathbf{5 0} \% \mathrm{~F} 12 \mathrm{Ham}$ 's cell culture medium with an N-2 neuronal cell growth supplement, was shown to support both a rate of growth, and the development of a range of features comparable to that which normally occur in vivo. Furthermore, the F12 (N-2) supplemented rat serum displayed a very low propensity to induce morphological abnormalities during the culture period. The study establishes a novel method of successful WEC using readily available commercial reagents and should enable the broader use of WEC.
\end{abstract}

KEY WORDS: rat serum, mouse embryo culture, F12, N2

\section{Introduction}

Embryology is dependent upon the ability to monitor the consequence of perturbed development. Perturbation can be induced by germ-line modification of an organism's genome or by physical manipulation of the embryo. The mouse has long been the vertebrate model organism of choice for genetic manipulation with techniques for transgenesis and targeted and random mutagenesis both long established and continually evolving. The combined effect of this is that alleles of every mouse gene will soon be available and the analysis of the associated phenotypes will continue to inform the study of the genetic control of mammalian development. In contrast to suitability for genetic manipulation, mammalian viviparity hinders observation following physical manipulation of post-implantation stage mouse embryos and experimental embryology approaches are most advanced in model organisms that reproduce externally such as fish and frog (Ellis-Hutchings and Carney 2010; Harris 2012; Hogan et al., 1994; Piliszek et al., 2011). Experimental embryology is however crucial to decipher embryonic development. Experiments in which cells are labelled and followed overtime generate the fate maps that underpin the study of an organism's development and transplantation experiments that transfer a group of cells from a donor to a host embryo reveal the inductive interactions that direct embryonic development (Hogan et al., 1994; Kaufman and Bard 1999). Combining these approaches with experimental embryology techniques provides a powerful means by which the consequence of germ-line modification can be analysed. To fully exploit mouse genetic resources, efficient and widely available protocols for the ex utero observation of murine embryos need to be developed.

The ex utero observation of murine development can achieved

\footnotetext{
Abbreviations used in this paper: CRSD, mixed culture medium comprising $50 \%$ commercial rat serum and 50\% DMEM cell culture medium; CRSDN, $50 \%$ commercial rat serum and 50\% DMEM with $\mathrm{N}-2$ neuronal cell growth supplement; CRSF, $50 \%$ commercial rat serum and 50\% F12 cell culture medium; CRSFN, 50\% commercial rat serum and $50 \% \mathrm{~F} 12$ with $\mathrm{N}-2$ neuronal cell growth supplement; DMEM, Dulbecco's Modified Eagle Medium; F12, Ham's F12 Nutrient Mixture, $\mathrm{N}-2$, neuronal cell growth supplement; WEC, whole embryo culture.
} *Address correspondence to: Ruth Arkell. Research School of Biology, Building 46, The Australian National University, Canberra, ACT, 0200, Australia.
Tel: +61-(0)2-6125-9158. e-mail: ruth.arkell @ anu.edu.au 
by whole embryo culture (WEC) and a range of studies have been conducted with this technique to investigate normal embryonic development (Downs and Gardner 1995; Ellington 1991; EllisHutchings and Carney 2010; Fleming et al., 1997; Foerst-Potts and Sadler 1997; Fujinaga 2000; Lawson et al., 1986, 1991; Lawson and Pedersen 1987; Moore-Scott et al., 2003; Morriss and New 1979; Nakagawa et al., 1997; Rivera-Perez et al., 2010; Tam 1989, 1998; Trainor et al., 1994; Wilson and Beddington 1996), to examine the effects of altering embryonic gene expression (Arkell and Beddington 1997; Fujinaga 2000; Pryor et al., 2012; Rivera-Perez et al., 2010; Takahashi et al., 2008; Tam 1998), or to screen chemical compounds for teratogenicity (Chatot et al., 1980; Ellis-Hutchings and Carney 2010; Harris 2012; Moore-Scott et al., 2003; Morriss and Steele 1974; Rivera-Perez et al., 2010; Tam 1998; Webster et al., 1997). The system that enables murine WEC was developed in the 1970's (Ellis-Hutchings and Carney 2010; New 1978; New et al., 1973; Tam 1998) and refined over the next two decades such that by the 1990's a standard protocol for WEC was established (Fujinaga 2000; Gray and Ross 2011; Harris 2012; Piliszek et al., 2011; Rivera-Perez et al., 2010; Takahashi and Osumi 2010). The method involves immersion of embryos in nutrient rich media, the supply of appropriate gas mixtures and rotation of embryos in the media at $37^{\circ} \mathrm{C}$. The technique itself is relatively straight forward, requiring no more expertise than the culture of primary or transformed cell lines. As with the culture of cell lines, serum is a crucial but ill-defined component of the nutrient rich media. In contrast to the media used for cell culture however, optimal mammalian WEC requires a high proportion (typically $50 \%$ or greater) of homologous serum (i.e. derived from the organism being cultured) and that serum collection avoid haemolysis (Hunter et al., 1988; Piliszek et al., 2011; Priscott 1983; Sadler and New 1981; Sanyal and Wiebke 1979; Sturm and Tam 1993; Tam 1998; Tam and Snow 1980).

The use of mouse serum for mouse embryo culture is problematic because of the small blood volume of the adult mouse: one mouse typically yields sufficient sera to culture two embryos. The development of murine WEC protocols therefore focused on achieving reasonable culture success using rat serum (since one rat typically yields sufficient sera to culture twenty embryos (Hunter et al., 1988)). Anumber of studies have shown that rat serum supports ex utero growth of post-implantation stage mouse embryos for at least 24 hours, providing that blood is withdrawn from the animal gently, immediately centrifuged, the fibrin clot carefully removed, and the sera heat-inactivated (Cockroft 1991; Harris 2012; New 1978; New et al., 1976; Piliszek et al., 2011; Rivera-Perez et al., 2010; Steele and New 1974; Takahashi and Osumi 2010; Tam 1998; Tam and Snow 1980; Van Maele-Fabry et al., 1991). The exacting requirements for the production of mouse embryo-culture-grade rat serum means commercial preparations are generally not able to support the ex utero growth of mouse embryos. Instead, current mouse WEC protocols recommend the preparation of rat serum 'in-house' as part of the embryo culture procedure (Fujinaga 2000; Harris 2012; Martin and Cockroft 2008; Piliszek et al., 2011; Pryor et al., 2012; Tam and Snow 1980).

The production of mouse embryo-culture-grade rat serum is not only technically demanding but is expensive and time consuming (Fujinaga 2000; Harris 2012; Martin and Cockroft 2008; RiveraPerez et al., 2010; Webster et al., 1997). The lack of a readily available, consistent source of rat serum hinders comparison between mouse WEC experiments and has constrained the use of this technique which has remained the preserve of a few specialised embryo laboratories world-wide. The current study aimed to develop a successful WEC medium that utilises only commercially produced reagents to support the ex utero growth of gastrula and neurula stage mouse embryos. Rat serum was commercially produced to custom instruction following the protocol known to be consistent with murine embryo culture. When combined with a standard cell culture medium (Ham's F12 Nutrient Mixture) and N-2 neuronal cell growth supplement the medium was found to support embryonic development that approximated in utero development and with the same frequency of success as previously published protocols that utilise in-house produced rat serum. The ability to purchase rat serum compatible with embryo culture should increase the number of researchers able to employ this technique, enable the routine coupling of murine experimental embryology with molecular genetics and likely drive further innovation in culture protocols.

\section{Experimental Protocols}

\section{Rat serum preparation}

Commercial rat serum was collected and processed by Valley Biomedical (Winchester, VA, USA catalogue number AS3061 with custom collection instructions) under sterile conditions. Rats were anaesthetised using $\mathrm{CO}_{2}$ and exsanguinated. Collected blood was immediately centrifuged at $1200 \mathrm{~g}$ for $10 \mathrm{~min}$, and the resulting fibrin clot squeezed and discarded. Individual bleeds were screened for haemolysis, and those selected for processing were re-centrifuged for a further $10 \mathrm{~min}$ at $1200 \mathrm{~g}$. The resulting serum layers were collected from the individual bleeds, pooled, aliquoted and frozen at $-20^{\circ} \mathrm{C}$.

\section{Culture media preparation}

Under sterile conditions, Ham's F12 Nutrient Mixture with Gluta$\max ^{\mathrm{TM}}$ (Invitrogen catalogue number 31765-035) was supplemented with N-2 neuronal cell growth supplement (100X, Invitrogen catalogue number 17502-048) at a concentration of $500 \mu \mathrm{L}$ per $50 \mathrm{~mL}$ of medium, (referred to here as F12 (N-2)). Then either Dulbecco's Modified Eagle Medium (supplemented with fresh L-glutamine $(2 \mathrm{mM})$ ) (Invitrogen, catalogue number 11995-073) or F12 (N-2) medium was combined with rat serum at a ratio of 1:1 to produce two distinct media referred to as Commercial Rat Serum DMEM (CRSD) or Commercial Rat Serum F12 (N-2) (CRSFN). For each stage and media condition tested a minimum of 18 embryos was scored and data combined from culture on at least five separate occasions. On some occasions, using the same basic formula of $50 \%$ rat serum and $50 \%$ medium, DMEM supplemented with $\mathrm{N}-2$ neuronal cell growth supplement at a concentration of $500 \mu \mathrm{L}$ per $50 \mathrm{~mL}$ of medium was used to generate Commercial Rat Serum DMEM N-2(CRSDN) medium whilst F12 medium not supplemented with N-2 was used to generate Commercial Rat Serum F12 (CRSF) medium. For each of these media a minimum of 12 embryos was cultured and data combined from culture on at least two separate occasions. Culture medium was filter sterilised through a $0.2 \mu \mathrm{m}$ filter into a $15 \mathrm{~mL}$ loosely-capped tube and allowed to equilibrate for at least $30 \mathrm{~min}$ at $37^{\circ} \mathrm{C}, 5 \% \mathrm{CO}_{2}$ in air in a sterile incubator.

\section{Embryo dissection}

Mice were maintained in a light cycle of 12 hours light: 12 hours 
dark, with the midpoint of the dark cycle occurring at 12 a.m. and according to Australian Standards for Animal Care under protocols approved by The Australian National University Animal Ethics and Experimentation Committee. To generate staged, genetically identical embryos for culture, female BALB/c animals were paired with C57BL/6 male animals and midday on the day of appearance of a vaginal plug was designated as 0.5 days post coitum (dpc). At the appropriate gestation point the CB6F1 embryos were dissected from the deciduum and free of maternal membranes under sterile conditions in $10 \%$ foetal bovine serum in PBS. Only embryos in which Reichert's membrane was resected without causing damage to either the embryo itself or to the trophectoderm were used for culture (Gray and Ross 2011). These embryos were staged (for pre-somite embryos according to Downs and Davies (Downs and Davies 1993), or by somite number), immersed in $500 \mu \mathrm{L}-1 \mathrm{~mL}$ of equilibrated culture medium in a sterile glass embryo dish, and held in a sterile $37^{\circ} \mathrm{C}, 5 \% \mathrm{CO}_{2}$ incubator.

\section{Embryo culture}

Maintaining sterile conditions, the embryos for culture were separated into groups of up to 4 stage-matched embryos (Downs and Davies 1993) and transferred to $2 \mathrm{~mL}$ of equilibrated culture medium in a sterile glass vial (BTC engineering, Cambridge, UK) and placed on a roller culture apparatus (BTC engineering, Cambridge, UK) pre-warmed to $37^{\circ} \mathrm{C}$. Premixed $5 \% \mathrm{CO}_{2}$ in air was supplied to the embryos at a constant rate (gas release from the bottle was at a pressure of $3-5 \mathrm{psi}$ ). After 24 hours, embryos were dissected in $10 \%$ Foetal Bovine Serum (FBS) in PBS $\left(4^{\circ} \mathrm{C}\right)$ and examined and photographed with a stereomicroscope (Nikon SMZ1500 microscope and DS-Ri1 camera). Each embryo was staged and morphologically assessed for stage-dependent characteristics such as yolk-sac circulation, headfold development, heart rate, embryo turning (flexion), somite development, neural tube closure and forebrain elongation (see developmental and deformity scoring schemes in supplementary materials). For each characteristic scored a theoretical maximum was set and the score for each individual embryo expressed as a fraction of the maximum possible score. These individual embryo scores were averaged across all embryos for each characteristic and presented as the mean \pm S.E.M. Within a developmental stage each embryo's score for the relevant characteristics (either developmental or deformity) were summed to give a developmental and deformity score for each embryo. The total scores for each embryo were averaged and presented as the mean \pm S.E.M. These final scores were analysed via a 2 tailed $t$ test to identify differences between embryos cultured in different media..

\section{Results and Discussion}

\section{Commercial rat serum supports culture of post-implantation embryos when combined with F12 (N-2)}

To determine whether the commercially produced rat serum is of sufficient grade to support the WEC of post-implantation stage mouse embryos it was used to produce two distinct media. Previous studies recommend DMEM or F12 as optimal cell culture media for combination with rat serum in a mixed embryo culture medium (Piliszek et al., 2011; Rivera-Perez et al., 2010), therefore the commercially produced rat serum was first used here as a direct substitute for in-house rat serum in a standard DMEM-based embryo culture medium (50\% rat serum, 50\% DMEM and termed CRSD). Second, it was used to produce an F12-based media that was modified by addition of an N-2 neuronal supplement $(50 \%$ rat serum, $50 \% \mathrm{~F} 12(\mathrm{~N}-2)$ and termed CRSFN). F12 is routinely used in combination with $\mathrm{N}-2$ neuronal cell growth supplement for applications such as neural crest cell culture (Garcia-Castro et al., 2002) and primitive streak culture (R. Arkell, personal observation). The planned culture experiments span the periods of primitive streak development and neurulation and it is therefore possible that $\mathrm{N}-2$ supplement will enhance embryo culture outcomes. In order to compare the relative efficacy of these two media, embryos (dissected at $7.5 \mathrm{dpc}$ or $8.5 \mathrm{dpc}$ ) were cultured for 24 hours under otherwise equivalent conditions and then examined for a variety of morphological criteria. As shown in Fig. 1 embryos dissected

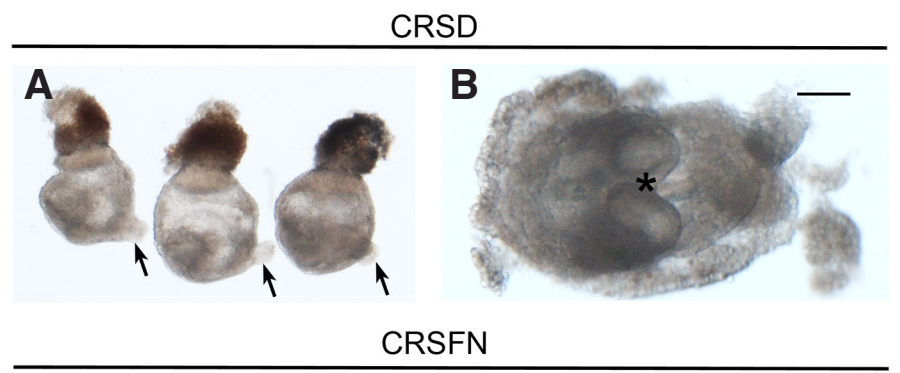

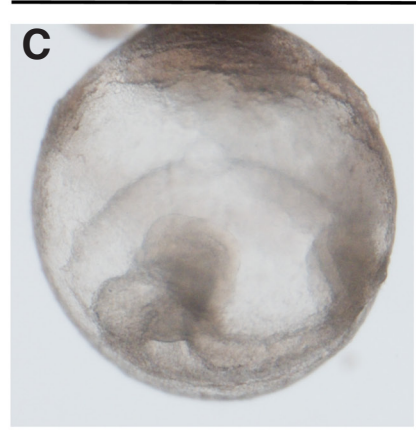

CRSD

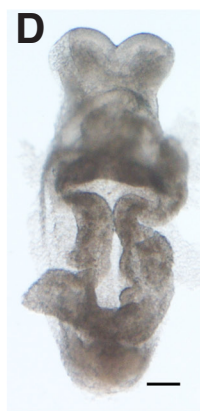

G

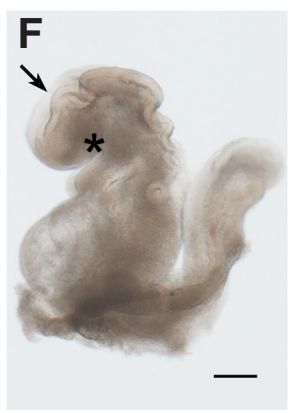

G

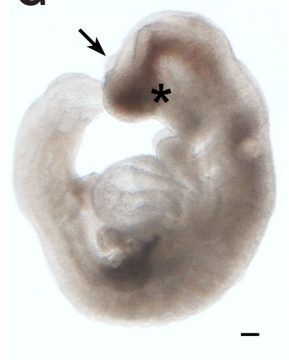

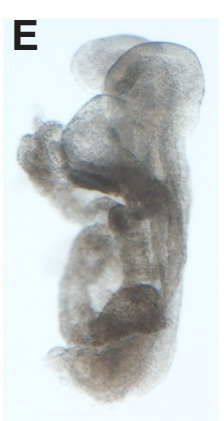

CRSFN

H

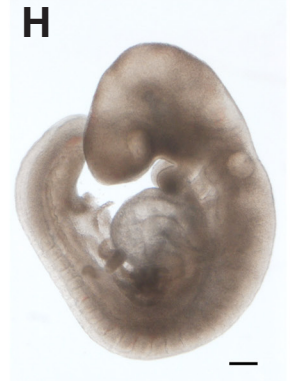

Fig. 1. Commercial rat serum can support whole embryo culture of 7.5 and 8.5 dpc murine embryos. Photographs of embryos following 24 hours of ex utero culture in each medium are shown, both with their extraembryonic membranes intact $(\mathbf{A}, \mathbf{C})$ or following membrane removal (B, D-H). Embryos are shown in lateral $(A, C, E-H)$ dorsal (B) or ventral (D) view, with anterior to the left $(A-C, E-H)$, or to the top (D). Culture was initiated with late primitive streak stage $(7.5 \mathrm{dpc})$ embryos (A-E) or with 6-somite stage $(8.5 \mathrm{dpc}$ ) embryos (F-H). Arrows in (A), allantois protruding from yolk sac; * in $(B)$, poorly developed head-folds; arrows in $(F, G)$, incomplete cranial neural tube closure; * in $(F, G)$, poor optic cup development. Scale bar, $100 \mu \mathrm{m}$. 
at either 7.5 or $8.5 \mathrm{dpc}$ and cultured in CRSD had overt signs of abnormal development, whereas those cultured in CRSFN appeared to develop to the equivalent in utero stage. Embryos that are grown in culture for 24 hours from the late primitive streak stage $(7.5 \mathrm{dpc})$ are expected to develop $\sim 6$ somites and to exhibit morphological landmarks of cranial neural development (Downs and Davies 1993; Kaufman and Bard 1999; Rivera-Perez et al., 2010; Takahashi and Osumi 2010; Van Maele-Fabry et al., 1993). The majority of embryos cultured in CRSD did not develop somites and their allantois often protruded through the yolk sac. In contrast, embryos grown in CRSFN appeared to develop as expected for their in utero counterparts (Fig. 1 A-E). Similar results were obtained for embryos cultured for 24 hours from the 6-somite stage. During the culture period, these embryos are expected to add a further 12 somites, to have complete embryonic rotation and cranial neural tube closure, and to develop optic and otic placodes, limb buds and branchial arches (Downs and Davies 1993; Kaufman and Bard 1999; Van Maele-Fabry et al., 1993). The majority of embryos cultured in CRSD did not meet all of these milestones and in fact $100 \%$ of these embryos exhibited one or more major deformities, most often including pyknosis (83\% of embryos) and forebrain truncation ( $87 \%$ of embryos), suggesting that their development was grossly impaired. In contrast, embryos grown in CRSFN appeared to develop as expected for their in utero counterparts and only $19 \%$ of embryos exhibited one or more major deformities (Fig. $1 \mathrm{~F}-\mathrm{H}$ ).

To quantify and compare the success of culture with either medium each embryo was evaluated to ascertain whether their rate of growth and development was as expected for the stage at which the embryos were placed into culture. Published scoring systems did not match the precise range of developmental stages examined here and a modified scoring system was developed (Harris 2012;

TABLE 1

\section{GROWTH AND DEVELOPMENT OF EMBRYOS} CULTURED FROM 7.5 DPCa

\begin{tabular}{lccc} 
& \multicolumn{2}{c}{ Culture media } & Max. possible \\
\cline { 2 - 3 } Feature & CRSD & CRSFN & score \\
\hline Developmental score & & & \\
Fraction of expected somite pairs added & $0.00 \pm 0.00$ & $1.00 \pm 0.00$ & 1.00 \\
Presence of heart & $0.05 \pm 0.05$ & $1.00 \pm 0.00$ & 1.00 \\
Integrity of yolk sac & $0.00 \pm 0.00$ & $1.00 \pm 0.00$ & 1.00 \\
Integrity of allantois & $0.43 \pm 0.11$ & $1.00 \pm 0.00$ & 1.00 \\
Forebrain development (presence of F/M & $0.24 \pm 0.07$ & $1.00 \pm 0.00$ & 1.00 \\
junction/neural folds) & & & \\
Midbrain development (presence of pre-otic sulcus) & $0.00 \pm 0.00$ & $1.00 \pm 0.00$ & 1.00 \\
Hindbrain development (presence of otic sulcus) & $0.00 \pm 0.00$ & $1.00 \pm 0.00$ & 1.00 \\
Total score & $0.68 \pm 0.20$ & $7.00 \pm 0.00^{\mathrm{b}}$ & 7.00 \\
& & & \\
Major Deformity score & & & \\
Pyknosis & $1.00 \pm 0.00$ & $0.00 \pm 0.00$ & 1.00 \\
Protrusion of allantois from yolk sac & $0.21 \pm 0.10$ & $0.00 \pm 0.00$ & 1.00 \\
Head/tail fusion & $0.95 \pm 0.05$ & $0.00 \pm 0.00$ & 1.00 \\
Total score & $2.16 \pm 0.12$ & $0.00 \pm 0.00^{\mathrm{C}}$ & 3.00 \\
\hline
\end{tabular}

a7.5 dpc embryos were cultured for approximately 24 hours in either CRSD $(n=20)$ or CRSFN $(n=$ 18) media. Each embryo was scored for rate of growth and the development of stage-appropriate morphology (developmental score) and for the presence of induced morphological abnormalities (major deformity score). A developmental score of 1.00 indicates that the embryo developed the given feature at the expected rate and with correct morphology. A major deformity score of 1.00 denotes the presence of an abnormal feature. Data were pooled for all embryos within each media group and are presented as the mean \pm S.E.M. ${ }^{b}$ The mean total developmental score of embryos cultured in CRSFN media was significantly higher than that of embryos cultured in CRSD media, $\mathrm{P}$ $<0.001$ ( 2 tailed $t$ test). ${ }^{C}$ The mean total major deformity score of embryos cultured in CRSFN media was significantly lower than that of embryos cultured in CRSD media, $\mathrm{P}<0.001$ ( 2 tailed $t$ test).
Van Maele-Fabry et al., 1990). For each culture period a range of morphological features was scored, consistent with an embryo of the stage equivalent to 24 hours after explantation (Tables 1 and 2). The score for these individual features were summed to give a 'developmental' score. Additionally, a range of stage appropriate features generally associated with poor embryonic development were scored and summed to generate a 'major' and a 'minor deformity' score (Tables 1 and 2) indicative of induced morphological abnormalities. Deformities severe enough to potentially confound experimental results or to necessitate exclusion of an embryo from a data set following WEC were considered major, whereas abnormalities that did not indicate impaired viability or significant growth retardation were considered minor (see supplementary material for a full description of the scoring system). Successful embryo culture is therefore associated with a high developmental score and a low deformity score. Embryos grown in CRSFN media accumulated significantly higher developmental scores than those cultured in CRSD for both age groups. Furthermore, CRSFN cultured embryos of both age groups scored significantly lower in terms of induced major morphological abnormalities. These results indicate that the F12 (N-2) based culture medium was better able to support both a normal rate of embryonic growth, and the development of expected features and was also less prone to inducing aberrant features than the DMEM based culture medium.

To measure the overall culture success of the CRSFN medium the proportion of embryos that exhibited a maximum developmental score and minimum major deformity score was calculated. For those embryos cultured from $7.5 \mathrm{dpc}$ for a 24 hour period $100 \%$ of embryos achieved this score. For those embryos cultured from 8.0 or $8.5 \mathrm{dpc}$ for a 24 hour period $81 \%$ of embryos received a maximum developmental score and minimum major deformity score. Pooling the data across all age groups, an overall culture success rate of $91 \%(n=49)$ was obtained. The overall culture success rate with the CRSFN media compares favourably with that reported in studies which utilize in-house prepared rat serum. For example, previous studies report a $70-80 \%$ success rate for murine embryos cultured from $7.5 \mathrm{dpc}$ for a 24 hour period and a success rate of $85-90 \%$ for embryos cultured from early somite stages for a 24 hour period (Arkell and Beddington 1997; Quinlan et al., 1995; Tam 1998; Trainor et al., 1994). Likewise, a recent study recommended a success rate of greater than $80 \%$ be indicative of a good WEC system for embryos cultured from $7.5 \mathrm{dpc}$ (RiveraPerez et al., 2010). It is possible that a low background rate of culture failure persists even under optimal WEC conditions due to damage unconsciously incurred during the dissection/explantation experimental procedures (Klug 1991). It therefore appears that commercially available rat serum prepared specifically for embryo culture can support ex utero growth of murine gastrula and neurula stage embryos.

\section{The difference in culture success of the two media is not determined by $\mathrm{N}-2$ alone}

The results presented above, in which the $\mathrm{F} 12(\mathrm{~N}-2)$ medium combined with commercial rat serum gave superior culture outcomes, could be explained entirely by the presence of the $\mathrm{N}-2$ neuronal cell growth supplement, which to our knowledge has not previously been used in the culture of gastrulating and neurulating murine embryos. If this is correct then the addition of $\mathrm{N}-2$ to the DMEM based medium would rectify the culture-induced defects 
associated with this medium and the removal of $\mathrm{N}-2$ from the $\mathrm{F} 12$ $(\mathrm{N}-2)$ based medium would render this medium incompatible with high rates of culture success. The effect of a 24 hour period of culture of $8.5 \mathrm{dpc}$ embryos in $50 \%$ commercial rat serum, 50\% DMEM (N-2) (referred to as CRSDN medium), and in 50\% commercial rat serum, $50 \% \mathrm{~F} 12$ (referred to as CRSF medium) was therefore examined. Visual inspection indicated that the CRSDN cultured embryos were only marginally improved from those grown in unsupplemented DMEM, exhibiting growth retardation and failures of neural tube closure but with reduced pyknosis and headfold wrinkling (Fig. 2A). Likewise, embryos grown in the non $\mathrm{N}$-2 supplemented CRSF culture medium showed growth and development that was considered approximately equivalent to that produced by culture in F12(N-2) (CRSFN). Notably, however, embryos grown in this (CRSF) medium had branchial arches that did not fuse at the ventral midline of the embryo, resulting in an open branchial pouch which was accompanied by an abnormal widening of the embryonic anterior despite anterior neural tube closure (Fig. 2B). This defect was observed in $75 \%$ of CRSF cultured embryos ( $\mathrm{n}$ $=12$ ). The origin of this defect is unclear, however it may involve neural crest cell migration since, though infrequently noted in CRSFN embryos, it appears to have been largely induced in the absence of the N-2 neuronal supplement from this media. Since $\mathrm{N}-2$ supplementation of the DMEM based medium did not produce

TABLE 2

\section{GROWTH AND DEVELOPMENT OF EMBRYOS CULTURED FROM 8.0/8.5 DPCa}

\begin{tabular}{lccc} 
& \multicolumn{2}{c}{ Culture media } & Max. possible \\
\cline { 2 - 3 } Feature & CRSD & CRSFN & score \\
\hline Developmental score & & & \\
Yolk-sac circulation & $1.00 \pm 0.00$ & $1.00 \pm 0.00$ & 1.00 \\
Fraction of expected somite pairs added & $0.70 \pm 0.05$ & $0.98 \pm 0.01$ & 1.00 \\
Presence of heartbeat & $0.83 \pm 0.08$ & $1.00 \pm 0.00$ & 1.00 \\
Presence of eyes & $0.26 \pm 0.09$ & $1.00 \pm 0.00$ & 1.00 \\
Fraction of expected branchial arches added & $0.70 \pm 0.07$ & $0.99 \pm 0.01$ & 1.00 \\
Extent of expected neural tube closure achieved & $0.26 \pm 0.09$ & $0.94 \pm 0.03$ & 1.00 \\
Extent of expected embryonic rotation achieved & $0.45 \pm 0.10$ & $0.92 \pm 0.04$ & 1.00 \\
Presence of expected forelimb buds & $0.87 \pm 0.07$ & $0.88 \pm 0.06$ & 1.00 \\
Total score & $5.06 \pm 0.42$ & $7.71 \pm 0.10^{\mathrm{b}}$ & 8.00 \\
Major Deformity score & & & \\
Head truncation & & & \\
Wrinkled headfolds & $0.87 \pm 0.07$ & $0.15 \pm 0.07$ & 1.00 \\
Pyknosis & $0.61 \pm 0.10$ & $0.08 \pm 0.05$ & 1.00 \\
Total score & $0.83 \pm 0.08$ & $0.08 \pm 0.05$ & 1.00 \\
Minor Deformity score & $2.30 \pm 0.15$ & $0.31 \pm 0.13^{\mathrm{c}}$ & 3.00 \\
Blood spotting & & & \\
Blebbing & & & \\
Total score & $0.01 \pm 0.00$ & $0.05 \pm 0.01$ & 0.10 \\
\hline & $0.03 \pm 0.01$ & $0.02 \pm 0.00$ & 0.10 \\
& $0.04 \pm 0.01$ & $0.07 \pm 0.01^{\mathrm{d}}$ & 0.20 \\
\hline
\end{tabular}

a $8.0 \mathrm{dpc}$ and $8.5 \mathrm{dpc}$ embryos were cultured for approximately 24 hours in either CRSD ( $n=23)$ or CRSFN $(n=26)$ media. Each embryo was scored for rate of growth and the development of stage-appropriate morphology (developmental score) and for the presence of induced morphological abnormalities (major deformity score and minor deformity score). A developmental score of 1.00 indicates that the embryo developed the given feature at the expected rate and with correct morphology. A major deformity score of 1.00 denotes the presence of a severe abnormality, while a minor deformity score of 0.10 indicates the presence of a much less significant abnormality. Data were pooled for all embryos within each media group and are presented as the mean \pm S.E.M. ${ }^{b}$ The mean total developmental score of embryos cultured in CRSFN media was significantly higher than that of embryos cultured in CRSD media, $\mathrm{P}<0.001$ ( 2 tailed $t$ test). ${ }^{\circ}$ The mean total major deformity score of embryos cultured in CRSFN media was significantly lower than that of embryos cultured in CRSD media, $\mathrm{P}<0.001$ ( 2 tailed $t$ test). ${ }^{\mathrm{d}}$ The mean total major deformity score of embryos cultured in CRSFN media was not significantly different to that of embryos cultured in CRSD media, $\mathrm{P}$ > 0.001 ( 2 tailed $t$ test). culture outcomes equivalent or superior to the CRSFN medium, these (CRSDN and CRSF) media combinations were not further pursued by quantification of culture outcomes. The results imply that $\mathrm{F} 12$ and $\mathrm{N}-2$ are together responsible for the improved culture outcomes associated with growth in 50\% commercial rat serum, $50 \%$ F12 (N-2) (CRSFN).

The finding that $\mathrm{N}-2$ supplementation improves WEC outcomes of gastrula and neurula embryos is perhaps not surprising given its known beneficial effects on the culture of primitive streak and developing neural tissues (Chiba et al., 2005; Haubensak et al., 2004; Pankratz et al., 2007). It has also been found to be advantageous in the culture of mid-gestation mouse embryos using serum free media (Moore-Scott et al., 2003). Failures of neural tube closure and poor forebrain elongation are often associated with WEC (Hunter et al., 1988; New et al., 1976; Sadler and New 1981; Tam and Snow 1980; Van Maele-Fabry et al., 1995) and it may be that $\mathrm{N}-2$ supplementation is a useful addition to any protocol for the WEC of post-implantation stage mouse embryos. It is however not clear why the use of F12 medium contributes to the improved culture outcome (relative to that with DMEM) when combined with the commercially produced rat serum. This is not generally the case for the culture of these stage embryos since a 1:1 combination of in-house produced rat serum with DMEM is the media combination most often recommended for murine WEC (Piliszek et al., 2011; Rivera-Perez et al., 2010; Tam 1998; Tam and Snow 1980; Van Maele-Fabry et al., 1995). A comparison of the components of the DMEM and F12 media used in this study shows that Phenol-Red is present at a ten-fold greater concentration in the DMEM. A systematic comparison of the consequence of Phenol Red on murine WEC has not been made but Phenol Red is known to act as a weak oestrogen and to have unexpected consequences on the culture of some mammalian cell lines (Berthois et al., 1986; Greiner et al., 2010; Lin et al., 2000). In support of the notion that commercially produced rat serum is sensitive to Phenol Red a recent description of murine WEC reports the use of commercial rat serum (Harlan Laboratories) in combination with Phenol Red free DMEM, but does not detail culture success rates (Gray and Ross 2011). Phenol Red serves as a pH indicator and

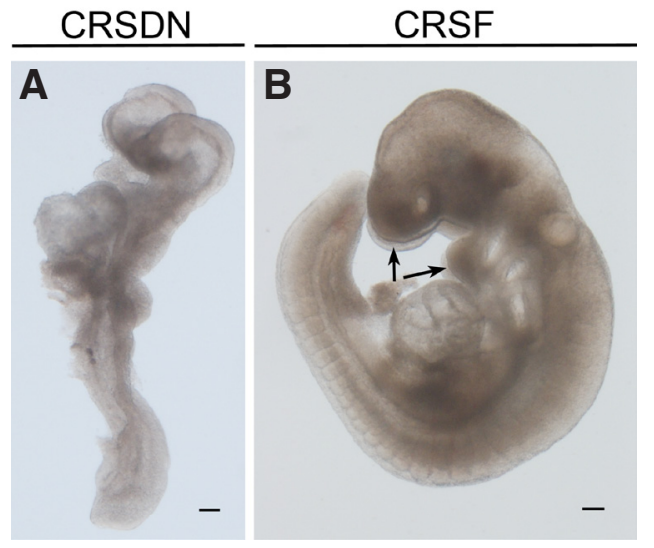

Fig. 2. N2 neuronal growth supplement does not fully account for the improved culture outcomes of the $\mathrm{F} 12(\mathrm{~N}-2)$ containing culture media. Lateral view of embryos cultured for 24 hours from the 6-somite stage $(8.5 \mathrm{dpc})$ in the media shown. Embryos are shown with anterior to the left. Arrows in (B): Incomplete branchial arch fusion and widened forebrain. Scale bar, $100 \mu \mathrm{m}$ 
therefore a marker of media exhaustion but the slow growth of cultured embryos and the limited culture period mean that this limit is rarely reached and that Phenol Red is dispensable for embryo culture. As with N-2 supplementation the use of Phenol Red free media may improve any murine embryo culture protocol.

The work presented here demonstrates that some companies are now willing and able to produce rat serum compatible with the WEC of murine embryos. To date we have received and tested three independent batches of rat serum from Valley Biomedical and have found all three useful for the culture of gastrula and neurula stage embryos. The ability to perform murine embryo culture with commercially available reagents should increase the number of laboratories that employ this technique and the frequency with which it is used. In addition, the ability to purchase (rather than make) mouse embryo-culture-grade rat serum should mean that researchers are more willing to dedicate it to experiments that seek only to compare the relative success of different culture media or other protocol modifications and so may drive much needed technical innovation in this area.

\section{Acknowledgements}

This work was supported by NHMRC grant 366476 (to R.M.A.). We acknowledge the technical assistance of Michael Zavortink and Philippa d'Ews Thomson whom established the whole embryo roller system in the Arkell laboratory and Drs Eldon Ball and Radiya Ali for critical reading of the manuscript.

\section{References}

ARKELL R, BEDDINGTON RS (1997). Bmp-7 influences pattern and growth of the developing hindbrain of mouse embryos. Development 124: 1-12.

BERTHOISY, KATZENELLENBOGENJA, KATZENELLENBOGENBS (1986). Phenol red in tissue culture media is a weak estrogen: Implications concerning the study of estrogen-responsive cells in culture. Proc Natl Acad Sci U S A 83: 2496-2500.

CHATOT CL, KLEIN NW, PIATEK J, PIERRO LJ (1980). Successful culture of rat embryos on human serum: Use in the detection of teratogens. Science 207: 1471-1473.

CHIBA S, KUROKAWA MS, YOSHIKAWA H, IKEDA R, TAKENO M, TADOKORO M, SEKINO H, HASHIMOTO T, SUZUKI N (2005). Noggin and basic fgf were implicated in forebrain fate and caudal fate, respectively, of the neural tube-like structures emerging in mouse es cell culture. Exp Brain Res 163: 86-99.

COCKROFT DL (1991). Culture media for postimplantation embryos. Reprod Toxicol 5: 223-228.

DOWNS KM, DAVIES T (1993). Staging of gastrulating mouse embryos by morphological landmarks in the dissecting microscope. Development 118: 1255-1266.

DOWNS KM, GARDNER RL (1995). An investigation into early placental ontogeny: Allantoic attachment to the chorion is selective and developmentally regulated. Development 121: 407-416.

ELLINGTON SK (1991). Use of embryo culture to study normal development and developmental mechanisms. Reprod Toxicol 5: 229-235.

ELLIS-HUTCHINGS RG, CARNEY EW (2010). Whole embryo culture: A "new" technique that enabled decades of mechanistic discoveries. Birth Defects Res $B$ Dev Reprod Toxicol 89: 304-312.

FLEMING A, GERRELLI D, GREENE ND, COPP AJ (1997). Mechanisms of normal and abnormal neurulation: Evidence from embryo culture studies. Int J Dev Biol 41: $199-212$.

FOERST-POTTS L, SADLERTW (1997). Disruption of msx-1 and msx-2 reveals roles for these genes in craniofacial, eye, and axial development. Dev Dyn 209: 70-84.

FUJINAGAM (2000). In vitro culture of rodent embryos during the early postimplantation period. Methods $\mathrm{Mol} \mathrm{Biol} 135$ : 53-76.

GARCIA-CASTRO MI, MARCELLE C, BRONNER-FRASER M (2002). Ectodermal wnt function as a neural crest inducer. Science 297: 848-851.
GRAY J, ROSS ME (2011). Neural tube closure in mouse whole embryo culture. J Vis Exp 56: e3132, doi:10.3791/3132

GREINERM, KREUTZERB, UNTEREGGERG, WULLICHB, ZIMMERMANNR(2010). Real-time analysis of Incap cell growth in different media. Biochemica 1: 14 - 15. HARRIS C (2012). Rodent whole embryo culture. Methods Mol Biol 889: 215-237.

HAUBENSAK W, ATTARDO A, DENK W, HUTTNER WB (2004). Neurons arise in the basal neuroepithelium of the early mammalian telencephalon: A major site of neurogenesis. Proc Natl Acad Sci U S A 101: 3196-3201.

HOGAN B, BEDDINGTON R, COSTANTINI F, LACY E (1994). Manipulating the mouse embryo. Cold Spring Harbour Press, New York.

HUNTER ES, BALKAN W, SADLER TW (1988). Improved growth and development of presomite mouse embryos in whole embryo culture. J Exp Zool 245: 264-269.

KAUFMAN MH (2002). The atlas of mouse development. Academic Press, London.

KAUFMAN MH, BARD JBL (1999). The anatomical basis of mouse development. Academic Press, San Diego.

KLUG S (1991). Whole embryo culture: Interpretation of abnormal development in vitro. Reprod Toxicol 5: 237-244.

LAWSON KA, MENESES JJ, PEDERSEN RA (1986). Cell fate and cell lineage in the endoderm of the presomite mouse embryo, studied with an intracellular tracer. Dev Biol 115: 325-339.

LAWSON KA, MENESES JJ, PEDERSEN RA (1991). Clonal analysis of epiblast fate during germ layer formation in the mouse embryo. Development 113: 891-911.

LAWSON KA, PEDERSEN RA (1987). Cell fate, morphogenetic movement and population kinetics of embryonic endoderm at the time of germ layer formation in the mouse. Development 101: 627-652.

LIN MF, LEE MS, GARCIA-ARENAS R, LIN FF (2000). Differential responsiveness of prostatic acid phosphatase and prostate-specific antigen mrna to androgen in prostate cancer cells. Cell Biol Int 24: 681-689.

MARTIN P, COCKROFT DL (2008). Culture of postimplantation mouse embryos. Methods Mol Biol 461: 7-22.

MOORE-SCOTT BA, GORDON J, BLACKBURN CC, CONDIE BG, MANLEY NR (2003). New serum-free in vitro culture technique for midgestation mouse embryos. Genesis 35: 164-168.

MORRISS GM, NEW DA (1979). Effect of oxygen concentration on morphogenesis of cranial neural folds and neural crest in cultured rat embryos. $J$ Embryol Exp Morphol 54: 17-35

MORRISS GM, STEELE CE (1974). The effect of excess vitamin a on the development of rat embryos in culture. J Embryol Exp Morphol 32: 505-514.

NAKAGAWAM, PRICE RL, CHINTANAWONGES C, SIMPSON DG, HORACEK MJ, BORG TK, TERRACIO L (1997). Analysis of heart development in cultured rat embryos. J Mol Cell Cardiol 29: 369-379.

NEW DA (1978). Whole-embryo culture and the study of mammalian embryos during organogenesis. Biol Rev Camb Philos Soc 53: 81-122.

NEW DA, COPPOLAPT, COCKROFT DL (1976). Improved development of head-fold rat embryos in culture resulting from low oxygen and modifications of the culture serum. J Reprod Fertil 48: 219-222.

NEW DA, COPPOLAPT, TERRY S (1973). Culture of explanted rat embryos in rotating tubes. J Reprod Fertil 35: 135-138.

PANKRATZ MT, LI XJ, LAVAUTE TM, LYONS EA, CHEN X, ZHANG SC (2007). Directed neural differentiation of human embryonic stem cells via an obligated primitive anterior stage. Stem Cells 25: 1511-1520.

PILISZEK A, KWON GS, HADJANTONAKIS AK (2011). Ex utero culture and live imaging of mouse embryos. Methods Mol Biol 770: 243-257.

PRISCOTT PK (1983). Rat post-implantation embryo culture using heterologous serum. Aust J Exp Biol Med Sci 61: 47-55

PRYOR SE, MASSA V, SAVERY D, GREENE ND, COPP AJ (2012). Convergent extension analysis in mouse whole embryo culture. Methods Mol Biol839: 133-146.

QUINLAN GA, WILLIAMS EA, TAN SS, TAM PP (1995). Neuroectodermal fate of epiblast cells in the distal region of the mouse egg cylinder: Implication for body plan organization during early embryogenesis. Development 121: 87-98.

RIVERA-PEREZ JA, JONES V, TAM PP (2010). Culture of whole mouse embryos at early postimplantation to organogenesis stages: Developmental staging and methods. Methods Enzymol 476: 185-203.

SADLER TW, NEW DA (1981). Culture of mouse embryos during neurulation. $J$ 
Embryol Exp Morphol 66: 109-116.

SANYALMK, WIEBKE EA (1979). Oxygen requirement for in vitrogrowth and differentiation of the rat conceptus during organogenesis phase of embryo development. Biol Reprod 20: 639-647.

STEELE CE, NEW DA (1974). Serum variants causing the formation of double hearts and other abnormalities in explanted rat embryos. J Embryol Exp Morphol 31: 707-719.

STURM K, TAM PP (1993). Isolation and culture of whole postimplantation embryos and germ layer derivatives. Methods Enzymol 225: 164-190.

TAKAHASHI M, NOMURA T, OSUMI N (2008). Transferring genes into cultured mammalian embryos by electroporation. Dev Growth Differ 50: 485-497.

TAKAHASHI M, OSUMI N (2010). The method of rodent whole embryo culture using the rotator-type bottle culture system. J Vis Exp 42: e2170, doi: 10.3791/2170

TAM PP (1989). Regionalisation of the mouse embryonic ectoderm: Allocation of prospective ectodermal tissues during gastrulation. Development 107: 55-67.

TAM PP (1998). Postimplantation mouse development: Whole embryo culture and micro-manipulation. Int J Dev Biol 42: 895-902.

TAM PP, SNOW MH (1980). The in vitro culture of primitive-streak-stage mouse embryos. J Embryol Exp Morphol 59: 131-143.
TRAINOR PA, TAN SS, TAM PP (1994). Cranial paraxial mesoderm: Regionalisation of cell fate and impact on craniofacial development in mouse embryos. Development 120: 2397-2408.

VANMAELE-FABRY G, DELHAISE F, GOFFLOTF, PICARD JJ (1993). Developmental table of the early mouse post-implantation embryo. Toxicol In vitro 7: 719-725.

VAN MAELE-FABRY G, DELHAISE F, PICARD JJ (1990). Morphogenesis and quantification of the development of post-implantation mouse embryos. Toxicol In vitro 4: 149-156.

VAN MAELE-FABRY G, GOFFLOT F, PICARD JJ (1995). Whole embryo culture of presomitic mouse embryos. Toxicol In vitro 9: 671-675.

VAN MAELE-FABRY G, PICARD JJ, ATTENON P, BERTHET P, DELHAISE F, GOVERS MJ, PETERS PW, PIERSMA AH, SCHMID BP, STADLER J, VERHOEF A, VERSEIL C (1991). Interlaboratory evaluation of three culture media for postimplantation rodent embryos. Reprod Toxicol 5: 417-426.

WEBSTER WS, BROWN-WOODMAN PD, RITCHIE HE (1997). A review of the contribution of whole embryo culture to the determination of hazard and risk in teratogenicity testing. Int J Dev Biol 41: 329-335.

WILSON V, BEDDINGTON RS (1996). Cell fate and morphogenetic movement in the late mouse primitive streak. Mech Dev 55: 79-89. 


\section{Further Related Reading, published previously in the Int. J. Dev. Biol.}

Postimplantation mouse development: whole embryo culture and micro-manipulation. P P Tam

Int. J. Dev. Biol. (1998) 42: 895 - 902.

Postimplantion mouse embryos cultured in vitro. Assessment with whole-mount immunostaining and in situ hybridisation.

G Van Maele-Fabry, F Clotman, F Gofflot, J Bosschaert and J J Picard

Int. J. Dev. Biol. (1997) 41: 365 - 374.

Mechanisms of normal and abnormal neurulation: evidence from embryo culture studies. A Fleming, D Gerrelli, N D Greene and A J Copp

Int. J. Dev. Biol. (1997) 41: $199-212$.

Genetic determinants of teratogen-induced abnormal development in mouse and rat embryos in vitro.

C M Kapron and D G Trasler

Int. J. Dev. Biol. (1997) 41: 337 - 344.

Surgical manipulation of mammalian embryos in vitro.

I Naruse, H Keino and M Taniguchi

Int. J. Dev. Biol. (1997) 41: 195 - 198.

5 yr ISI Impact Factor $(2011)=2.959$
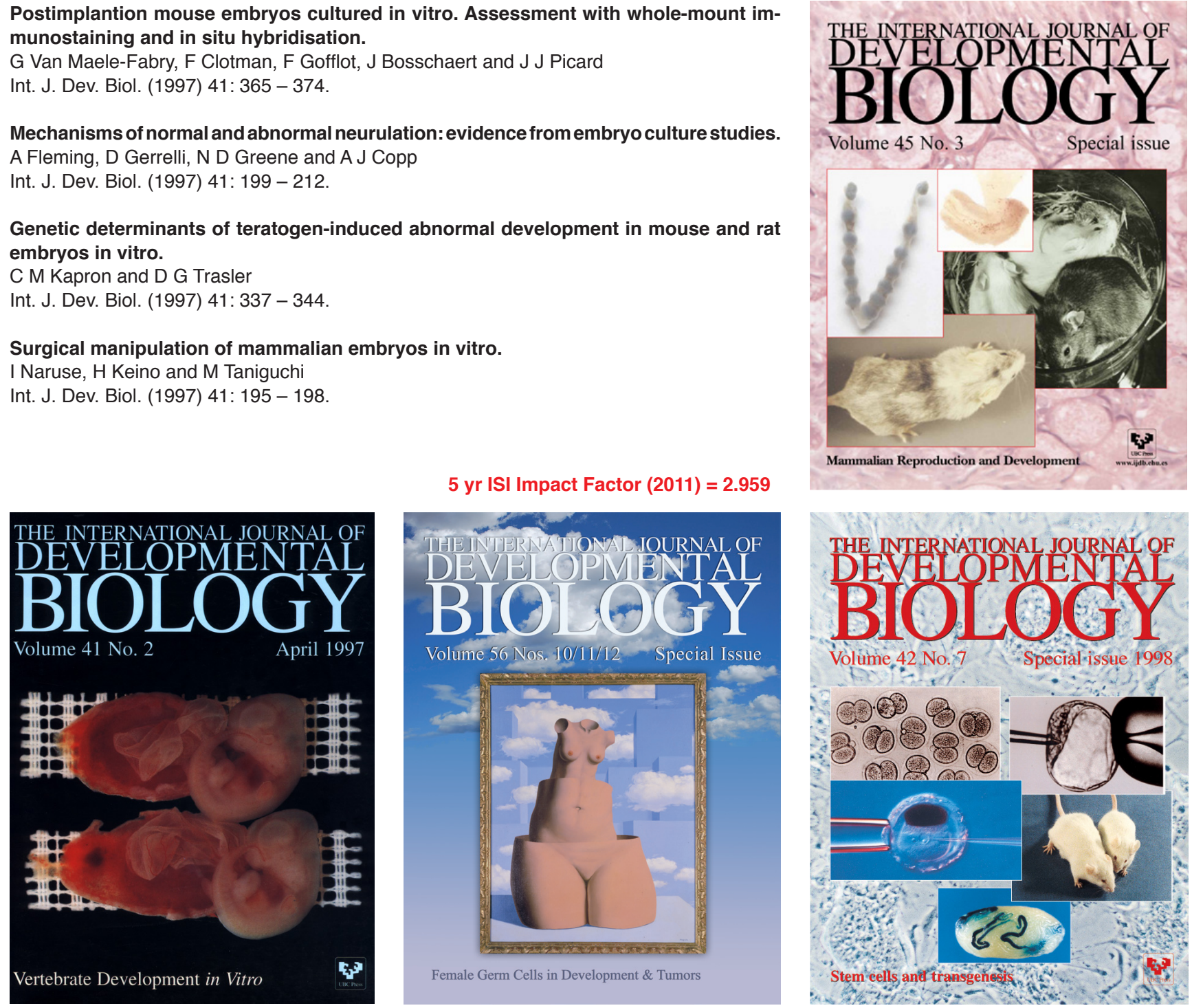